\title{
On the dynamics of Trojan planets in extrasolar planetary systems
}

\author{
R. Dvorak ${ }^{1}$, R. Schwarz ${ }^{2}$ and Ch. Lhotka ${ }^{1}$ \\ ${ }^{1}$ Institute for Astronomy, University of Vienna, \\ Türkenschanzstrasse 17, A-1180, Vienna, Austria \\ email: dvorak@astro.univie.ac.at, Ihotka@astro.univie.ac.at \\ ${ }^{2}$ Department of Astronomy, Eötvös University, \\ Pázmány Péter sétány 1/A, H-1117 Budapest, Hungary \\ email: schwarz@astro.univie.ac.at
}

\begin{abstract}
In this article we examine the motion of fictitious Trojan planets close to the equilateral Lagrangean equilibrium points in extrasolar planetary systems. Whether there exist stable motion in this area or not depends on the massratio of the primariy bodies in the restricted three body problem, namely the host star and the gasgiant. Taking into account also the eccentricity of the primaries we show via results of extensive numerical integrations that Trojan planets may survive only for $e<0.25$. We also show first results of a mapping in the 1:1 resonance with a gas giant on an eccentric orbit which is applied to the extrasolar planetary systems HD 17051. We furthermore study the influence of an additional outer planet which perturbs the motion of the gasgiant as well as the Trojan cloud around its $L_{4}$ Lagrangean point.
\end{abstract}

Keywords. Celestial mechanics, mapping, Trojan planets, HD 17051

\section{Introduction}

The search for terrestrial planets (=TP) in extrasolar planetary systems (=EPS) is one primary subject of scientists involved in this rapidly growing field of astronomy. Although up to now we have only knowledge of TPs with several Earth masses we hope to find quite soon a second Earth via space-based observations. It is the task of astrodynamical investigations to look for possibly stable orbits of additional planets in EPS where already one, or even more gas giants $(=\mathrm{GG})$ have been detected. Out of the different possible configurations for TPs, namely orbiting inside a GG (like in our Solar System), outside a GG (we know of many hot Jupiters) we also must take into account that a TP may have a GG with a TP as a satellite (e.g. Saturn's Titan). We concentrate in this study on coorbital TPs, which could be realized as Trojan TPs (=TTP).

There are several important studies on the Trojan problem: e.g. Marzari \& Scholl 1998, Nauenberg 2002, Laughlin \& Chambers 2002, Menou \& Tabachnik 2003, Morbidelli et al. 2005.

In chapter 2 we show the results of numerous integrations which have been undertaken to establish the largeness of the stable regions around the equilateral points of a large planet depending on the mass and the eccentricity of the primaries. We also show a mapping in the 1:1 resonance in the elliptic restricted three body problem, which gives a good indication of the dynamical structure of the Trojan region. In chapter 3 we concentrate on the perturbation of the stable region caused by a large planet outside the GG hosting the TP which mimics quite well the Solar system situation (e.g. Jupiter and Saturn). Finally, in the last chapter, we summarize the results which are interesting with 

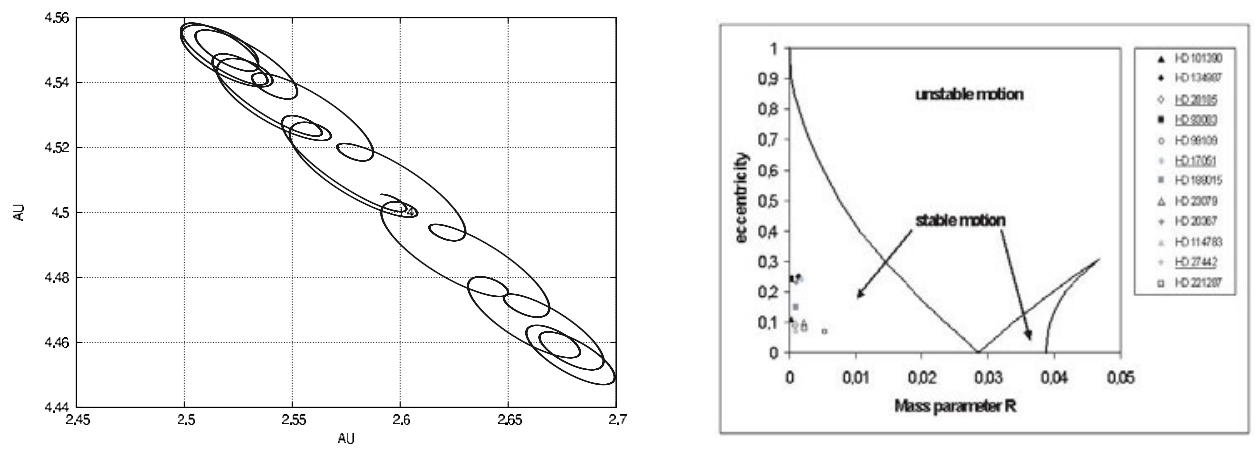

Figure 1. Typical motion of a Trojan asteroid in a rotating frame close to the equilateral equilibrium point $L_{4}$ (left graph). The stability of the equilateral equilibrium points depending on the mass ratio $\mathrm{P}$ and the eccentricity of the primary bodies after Marchal, 1990 (right graph).

respect to formation mechanism (Beaugé et al. 2007) and the chances to observe such TTPs (e.g. Ford \& Gaudi, 2006 and Goździewski \& Konacki 2007).

\section{The Trojan Regions in Extrasolar Planetary Systems}

It is known since the first discovery of an asteroid staying always in the vicinity of the Lagrangean equilibrium point (Fig. 1) that the work of Lagrange and Euler in the restricted three body problem $\dagger$ was not only of theoretical interest. This first asteroid was discovered by Max Wolf in Heidelberg in 1906 who named it after Achilles, the hero of the Trojan War. In the following years many more asteroids were discovered in Jupiter's Lagrangian points and were given names associated with the Iliadł.

\subsection{Numerical determination of the stable region for Trojans}

Part of the work was to find out how large is the extension of the stable regions around the equilateral Lagrangian points (Efthymiopoulos \& Sándor, 2005). From theory we know that the equilateral points are linearly stable even for large eccentricities of the primaries since the first work of Danby (1964). Fig. 1 shows zones of stability for motions of Trojan bodies in terms of the mass parameter $\mathrm{P}$ and the eccentricity. In addition we plotted the positions of all habitable extrasolar systems. The mass parameter $\mathrm{P}$ is defined through the equation (see Marchal, 1990):

$$
P=\frac{\left(m_{2}+m_{3}\right)}{M}+\frac{m_{2} \cdot m_{3}}{m_{1}^{2}}+O\left(m_{2}^{3} \cdot \frac{m_{3}}{m_{1}^{4}}\right)
$$

$\dagger$ A massless regarded planet is moving under the gravitational influence of Sun and a gasgiant like Jupiter in the same plane, where the two massive bodies, called the primies have circular orbit.

$\ddagger$ This group of asteroids is called 'Trojans'. The ones close to the L4 point are named after Greek heroes, the ones close to the L5 point are given names of the heroes of Troy. 617 Patroclus, a Greek warrior, is wrong placed as it is in the 'defending' Trojan camp, whereas 624 Hektor, a Trojan warrior, is in the Greek camp.

I Which means Extrasolar systems where one gas giant stays partly or fully in the habitable zone. 


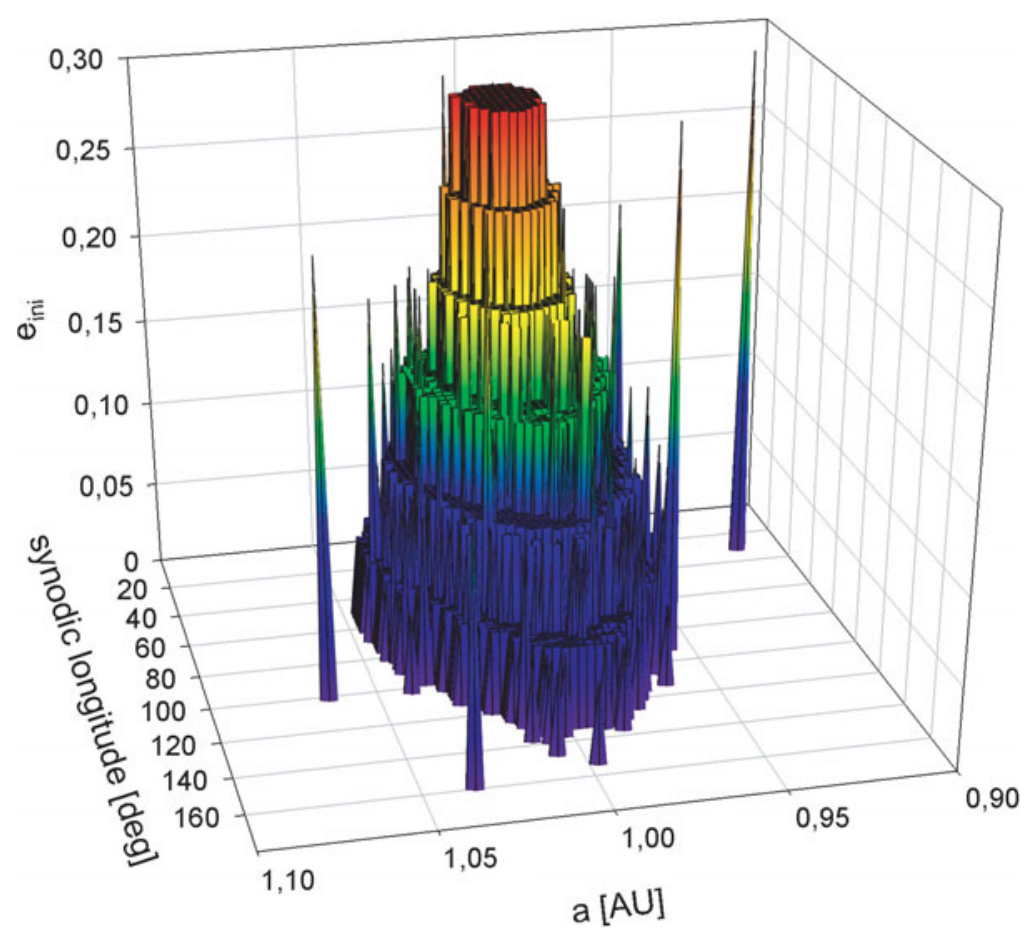

Figure 2. Stable area around the Lagrange point $L_{4}$ in the restricted three body problem for a dynamical model Sun-Jupiter depending on the eccentricity of the primaries.

$m_{1}, m_{2}, m_{3}$ are the masses of the star, the perturbing planet and the TTP respectively and $\mathrm{M}$ is the total mass of the system. Several studies have already been dedicated to this problem for extrasolar planetary systems (e.g. Dvorak and Schwarz 2005, Érdi and Sándor 2005, Érdi et al. 2007, Schwarz et al. 2005, 2007a and 2007b). The objectives of these investigations were to examine the dynamics of a terrestrial planet in 1:1 mean motion resonance with a Jovian-like planet. We show in Fig. 2 how the size of the Trojan stability regions for a Jupiter sized planet depends on the eccentricity of its orbit. This stable area was determined making use of the results of the integration of the equations of motion of a fine grid of initial conditions around the $L_{4}$ point for 1 million periods of the primaries. Finally a catalogue of hypothetical habitable Trojan planets (http://www.univie.ac.at/adg/) was computed, where the largeness of the stable region depending on the mass ratio of the star to the planet and of the eccentricity of the primaries' orbits is shown.

\subsection{Analytical mapping model for terrestrial trojan planets}

To obtain a mapping model for the 1:1 resonance of the elliptic restricted three body problem, we expanded the corresponding disturbing function $\mathrm{R}$ of the Hamiltonian (where $\mu=m_{2} /\left(m_{1}+m_{2}\right)$ is the mass ratio of the primaries (we assume $m_{1}>>m_{2}$ and set $\left.x=\sqrt{\frac{a}{a^{\prime}}}-1\right)$, see also Hajidemetriou 1993: 


$$
H=H_{0}+\mu H_{1}=-\frac{1}{2(1+x)^{2}}-(1+x)-\mu R,
$$

with respect to the eccentricities $\left(e, e^{\prime}\right)$ and the variation in the semi-major axis of the ratio between the axis of the asteroid $(a)$ and the disturbing planet $\left(a^{\prime}\right)$ to suitable high orders (' indicates the disturbing body).

The set of variable used to describe the systems are the Delaunay variables (Érdi \& Sándor, 2005) defined by the quantities:

$$
\tau=\lambda-\lambda^{\prime}, y=\sqrt{\frac{a}{a^{\prime}}}\left(\sqrt{1-e^{2}}-1\right), \omega,
$$

where $\lambda$ is the orbital longitude and $\omega$ means the argument of pericenter of the TTP. In the case of HD 17051 we carried out the expansion up to 7 th order in the eccentricites to cover the full domain of possible motions in the extra-solar Trojan configuration, such that the disturbing function turns out to be:

$$
R\left(\tau, \omega, x, y ; a^{\prime}, e^{\prime}\right)=\sum_{\nu} B_{\nu}\left(x, y ; a^{\prime}, e^{\prime}\right) \cos \Phi_{\nu}(\tau, \omega, \lambda),
$$

where $B_{\nu}$ are polynomial functions and $\Phi_{\nu}$ are the corresponding phase functions holding together terms of equal order $\nu$. A detailed description on the developement of the disturbing function can be found e.g. in (Hajidemetriou, 1993 and Lhotka et al., 2008). Using the method of Hadjidemetriou (1991) we setup the generating function:

$$
W=x_{n+1} \cdot \tau_{n}+y_{n+1} \cdot \omega_{n}+T \cdot \bar{H}\left(\tau_{n}, \omega_{n}, x_{n}, y_{n}, x_{n+1}, y_{n+1}\right),
$$

where $\bar{H}=H_{0}+\mu \bar{R}$ and $\bar{R}$ is the averaged disturbing function with respect to the orbital longitude of Jupiter $\lambda^{\prime}$ and $\mathrm{T}$ is the period of the mapping. The mapping model is derived via the equations

$$
J_{i, n}=\frac{\partial W}{\partial \theta_{i, n}}, \theta_{i, n+1}=\frac{\partial W}{\partial J_{i, n+1}}, i=1,2,
$$

where $J=(x, y)$ and $\theta=(\tau, \omega)$ and the mass parameter in $(2.2)$ is set equal to $\mu=$ 0.01795 .

The resulting mapping is defined on the 4 dimensional Poincaré surface of section, but given in its implicit form. Former studies (Lhotka et al. 2008) showed that the radius of convergence of the proposed mapping method becomes limited to the librational regime of the asteroid's motion, if we expand the generating function to make the mapping explicit by series reversion. Therefore we introduced a simple root-finding algorithm to iterate the mapping at each iteration step without expanding it into explicit form, so that we can preserve all possible dynamical behaviour of the mapping. For this reason we used the inital values $\left(\tau_{n}, \omega_{n}, x_{n}, y_{n}\right)$ not only as a starting point for the mapping iteration itself, but also as starting values for the root finding procedure to find $\left(\tau_{n+1}, \omega_{n+1}, x_{n+1}, y_{n+1}\right)$. In the respective figures we show two projections of the phase portrait, which is originally a 4 dimensional manifold in 4D phase space. Nevertheless the Poincaré surface of section $\left((\tau, \omega, x, y): \omega=\omega_{0}, \omega_{n+1}-\omega_{n}>0,(\tau, \omega, x, y): \tau=\tau_{0}, \tau_{n+1}-\tau_{n}>0, x_{n}>0\right)$ reveals the resonant structure of the system (chain of islands and the librational and rotational behaviour of the asteroid (Fig. 3)).

\section{Perturbations of a large planet on terrestrial trojan planets}

We know the extension of the stable regions in the elliptic restricted problem quite well; even when the third body has a small mass, that means in the general three body 

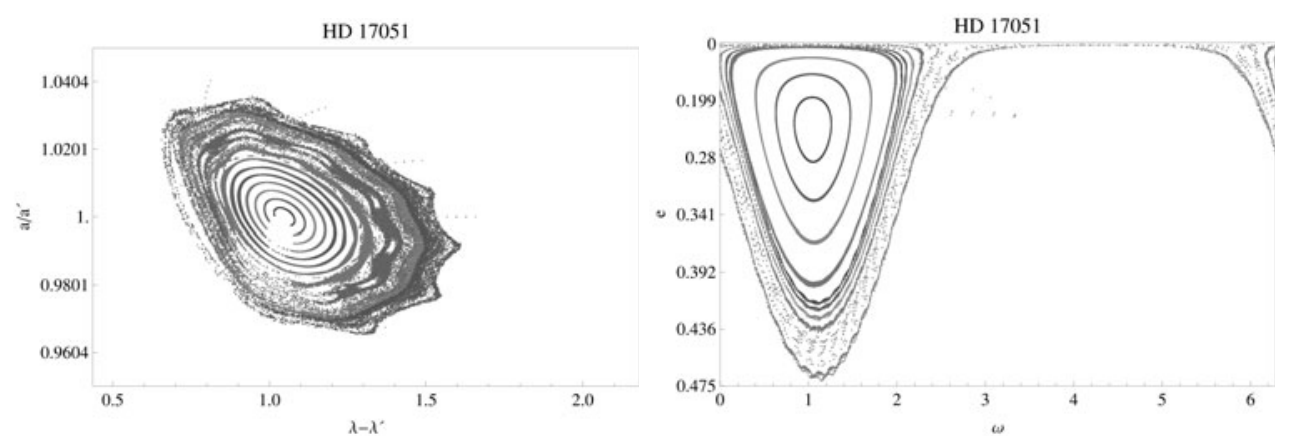

Figure 3. Mapping of the area around the Lagrange point $L_{4}$ in the system HD 17051: semimajor axis - libration angle (left graph) and eccentricity - pericenter (right graph).

problem there are stable areas. We now pose the question what happens when another large planet is perturbing these bodies, a situation comparable to a configuration in our Solar System, where Saturn perturbs the Jupiter Trojans. In this case, where the distance of Saturn is only twice the distance of Jupiter with both planets on low eccentric orbits, we have still a large population of Jupiter Trojans. Because nowadays we know already 25 EPS hosting more than one large planet, we study - as first approach to this question - how a second outer planet can disturb the Trojan region of an inner planet.

We put one Jupiter sized planet in a distance of $1 \mathrm{AU}$ and populated the $L_{4}$ region with massless Trojan bodies; a second large planet was included in our computations as an outer perturber in different distances $(1.2 A U<a<3.5 A U)$. The orbital eccentricities of both giants were set to equal values: $\mathrm{e}=0 ., 0.05,0.10,0.15$ and 0.20 . To be able to catch the most favorable and the most unfavorable initial configurations we investigated 8 different initial position (IP) for the two giants, which we call aligned (4 positions) and antialigned (4 positions) initial conditions for the inner and outer planet $\dagger \mathrm{P}$ (eriastron) $\mathrm{P}$ $(1,5), \mathrm{PA}$ (poastron $(2,6), \mathrm{AP}(3,7), 4$ : AA $(4,8)$; the numbers correspond to the $\mathrm{y}$-axis in Fig. 4 and Fig. 5. As integration method we used the mercury 6 symplectic integrator (Chambers, 1999) and the integration time was set to 1 million years for 100 fictitious Trojans around $L_{4}$. The stability criterion was a simple check of escape from that region, the largeness was defined as the number (percentage) of remaining Trojans after the end of the integration time. We need to emphasize that especially when we put the perturbing (outer) planet close to the inner planet, which hosts the Trojans, the mean motion resonances in connection with the different initial conditions are important for the stability of an orbit.

The discussion of the results leads to the following conclusions:

- $e=0$ and $e=0.05$ (Fig. 4, upper graph): We just show the results of the slightly eccentric orbits of the two planets because they are quite similar to the initially circular case. From $a_{2}=1.8 \mathrm{AU}$ on almost 40 orbits survived; there is only a slight decrease of the number of survivers for larger distances. The antialigned IP led to a smaller stable region.

- $e=0.10$ (Fig. 4, lower graph): For this moderate eccentricities the stable region $\dagger \mathrm{P}=$ Pericenter, $\mathrm{A}=$ Apocenter. 


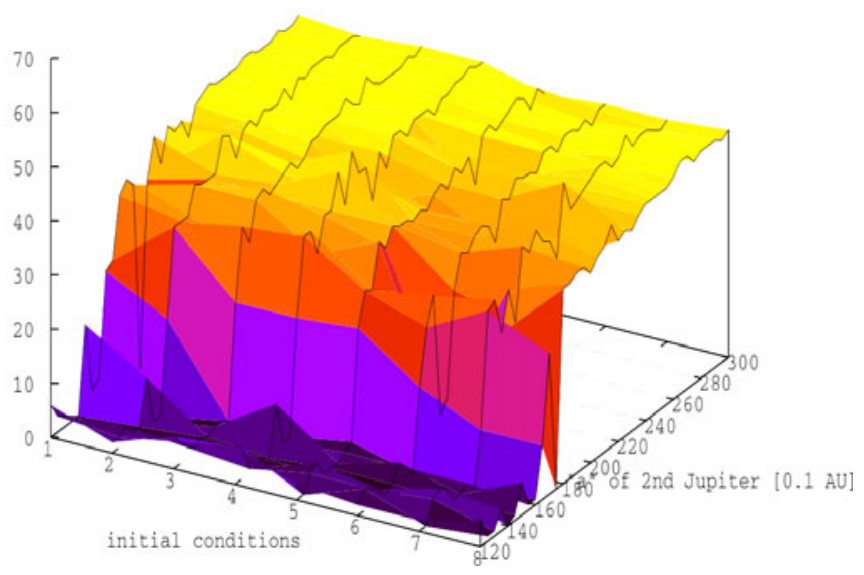

remaining orbits

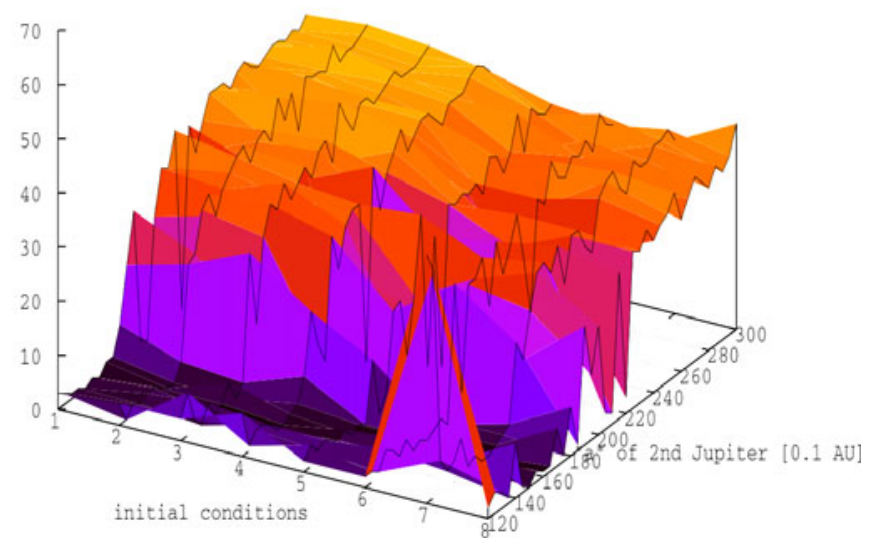

Figure 4. Initial condition diagram of the number of surviving Trojans (z-axes) around $L_{4}$ depending on the initial distance of the perturbing Jupiter (x-axis) and the position of the two planets (y-axes): $e=0.05$ (upper graph) and $e=0.10$ (lower graph) initial eccentricities of the two planets.

extends to a perturbing Jupiter as far as $a=1.8 A U$ and the the difference for aligned and antialigned IP starts to be significant.

- $e=0.15$ (Fig. 5, upper graph): for the aligned IP of the two primaries the difference to the former picture is not very large, but for the antialigned IP the unstable region extends to $2 \mathrm{AU}$ and globally the percentage of the surviving orbits close to $L_{4}$ is halved.

- $e=0.20$ (Fig. 5, lower graph): In both positions the unstable region is quite large (extends to $1.8 \mathrm{AU}$ ) for the aligned IP and up to $2.2 \mathrm{AU}$ for the antialigned IP, where almost no Trojans survived.

Already for $e=0.15$ and 0.20 one can see that even when the perturbing outer planet is more than two $A U$ from the Sun, the MMRs start to cut the stable region into different 'slices' of stable and unstable areas for most of the initial conditions. From $e=0.25$ of the inner Jupiter almost no stable Trojans survived independent of the perturbing outer Jupiter and thus we needed not to continue our investigations in this sense. 
remaining orbits

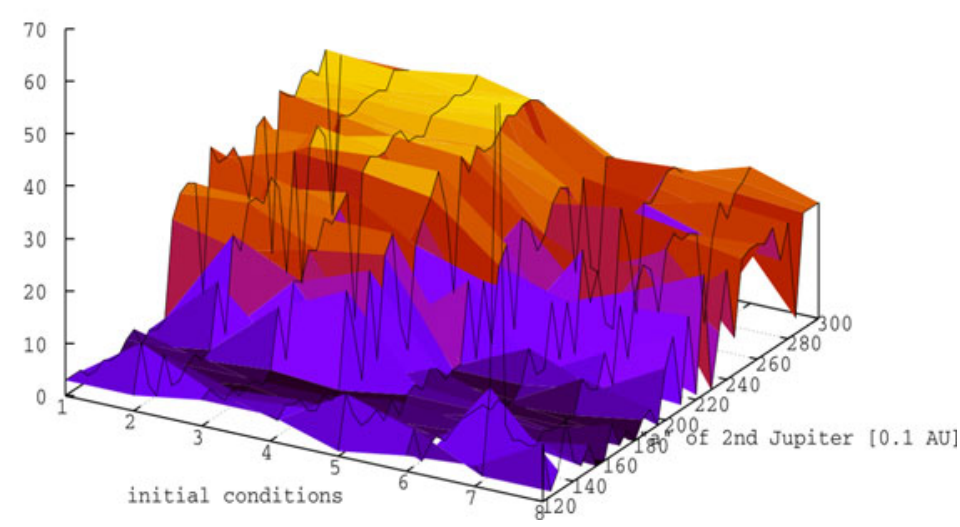

remaining orbits

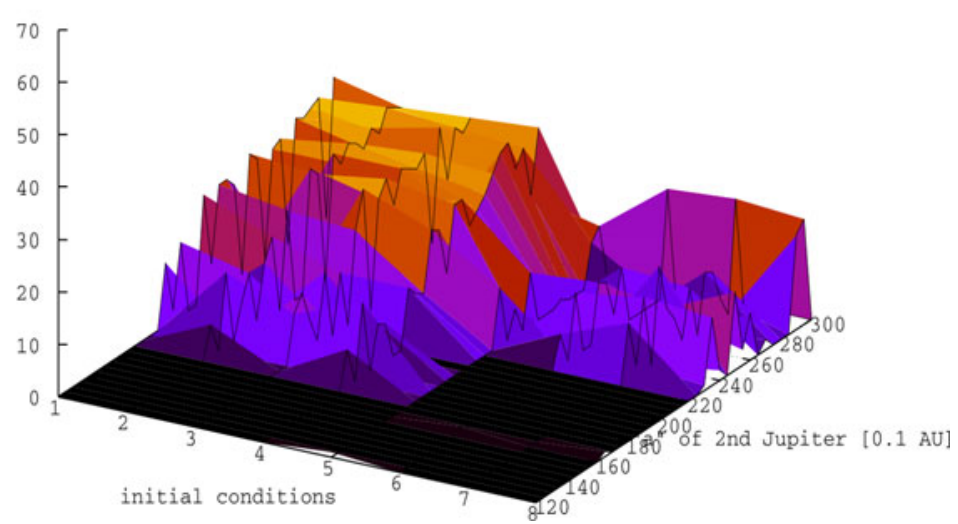

Figure 5. Caption like in Fig. 4 for $e=0.15$ (upper graph) and $e=0.20$ (lower graph).

\section{Conclusion}

We discussed how large the Trojan regions extends for single planet system depending on the eccentricity of the planet's orbit. In an analytical mapping model we also showed the structure of the stable region for the system HD 17051. Because more and more EPS with several large planets are observed we extended our investigations to an additional perturber outside the Trojan hosting large planet. The results show that - with a certain dependence on the eccentricities - the probability for the existence of stable Trojans of the inner large planet in EPS consisting of at least two large planets smaller than a ratio of the semimajor axes of $1: 2$ is quite large.

Acknowledgements: R. Schwarz thanks the Schrödinger grant of the FWF (J2619N16); the work of C.Lhotka was fully supported by the FWF project P-18930.

\section{References}

Beaugé, C., Sándor, Zs., Érdi, B., \& Süli, Á.: 2007, A\&A, 463, 359.

Chambers, J. E.: 1999, MNRAS, 304, 793. 
Danby, J. M. A.: 1964, AJ, 69, 165.

Dvorak, R. \& Schwarz, R.: 2005, CeMDA, 92, 19.

Efthymiopoulos C. \& Sándor, Z.,: 2005, MNRAS, 364, 253.

Érdi, B. \& Sándor, Z.: 2005, CeMDA, 92, 113.

Érdi, B., Nagy, I., Fróhlich, G., Sándor, Zs. and Súli, Á.: 2007b, MNRAS, 381, 33.

Ford, E. B. \& Gaudi, B. S.: 2006, ApJ, 652, 137.

Goździewski, K. \& Konacki, M.:2007, ApJ,647, 573.

Hadjidemetriou, J.: 1991, In A. E. Roy (Ed.), "Predictability, Stability and Chaos in N-Body Dynamical Systems', Plenum Press, 157.

Hajidemetriou, j.: 1993, CeMDA, 56, 563.

Lhotka Ch., Efthymiopolous, C. \& Dvorak, R.: 2008, MNRAS, 384, 1165.

Laughlin, G. \& Chambers, J. E.: 2002, AJ, 124, 592.

Marchal, C.: 1990, The three-Body Problem, Elsevier, 49.

Marzari, F. \& Scholl, H.: 1998, A\&A,339, 278.

Menou, K. \& Tabachnik, S.: 2003, AJ, 583, 473.

Morbidelli, A., Levison, H. F., Tsiganis, K., \& Gomes, R.: 2005, Nature,435, 462.

Nauenberg, M.: 2002, AJ., 124, 2332.

Schwarz, R., Pilat-Lohinger, E., Dvorak, R., Érdi, B., \& Sándor, Zs.: 2005, AsBio, 5, 579.

Schwarz, R., Dvorak, R.,Pilat-Lohinger, E., Süli, Á., \& Érdi, B.: 2007a, A\&̊A, 462, 1165.

Schwarz, R., Dvorak, R., Süli, Á., \& Érdi, B.: 2007b, A\&A, 474, 1023. 